\title{
Estudio de embarazo en adolescentes en 11 instituciones colombianas
}

\author{
Dres. Francisco Pardo V., Germán Uriza G.
}

\begin{tabular}{lll}
\hline \multicolumn{1}{c}{ NOMBRE } & INSTITUCION & CIUDAD \\
\hline & & \\
DARIOZAPATA & SANJUANDEDIOS & ARMENIA \\
ANTONIOLOMANTO & MATERNOINFANTIL & BOGOTA \\
ROBERTOJARAMILLO & SANJOSE & BOGOTA \\
GUILLERMOARBOLEDA & HOSPITALGENERAL & MEDELLIN \\
MANUELYANEZ & INSTITUTODE & \\
& SEGUROSSOCIALES & CUCUTA \\
LUCRECIAMOJICA & HOSPITALREGIONAL & VILLAVICENCIO \\
VICTOR RODRIGUEZ & SANIGNACIO & BOGOTA \\
MIGUEL RODRIGUEZ & SANRAFAEL & FACATATIVA \\
JAVIERMUÑOZ & DEPARTAMENTAL & PASTO \\
JOSEVICENTEERAZO & UNIVERSITARIO & POPAYAN \\
OSCARGOMEZ & RAMONGONZALEZVALENCIA & BUCARAMANGA \\
\hline
\end{tabular}

\section{Introducción}

En Colombia se ha venido trabajando en el campo de la salud reproductiva desde hace varios años siendo en la actualidad el área de la Adolescencia prioritaria para estudios que exploren la conducta sexual de nuestros jóvenes y el aumento cada vez mayor del embarazo en esta época de la vida.

En el aumento del embarazo en la adolescencia se encuentra la combinación de tres factores a saber: 1) Inicio temprano de las relaciones sexuales $(3,5,7,11)$. 2) Maduración sexual más temprana $(1,3,5,6,11)$. 3) Poca utilización de la anticoncepción preventiva por ignorancia o inaccesibilidad a los anticonceptivos $(3,5,11)$.

El embarazo a esta temprana edad es en su mayoría indeseado, ocurre frecuentemente en jóvenes solteras $(7,11$, 12) y es considerado desde el punto de vista obstétrico como un embarazo de alto riesgo por sus múltiples implicaciones sociales y médicas $(1,3,7,10,11)$.

El embarazo en la adolescencia y sus consecuencias como el aborto, la cesión del hijo, el madresolterismo y el hijo indeseado, así como las medidas tendientes a su prevención como la educación sexual y la anticoncepción preventiva, son temas prioritarios de la especialidad en Ginecología y Obstetricia y para las entidades gubernamentales que manejan la salud de la madre y del niño en cualquier país del mundo.

La literatura colombiana sobre el adolescente es muy extensa como lo demuestra la recopilación bibliográfica con 1.199 referencias, publicada por la Asociación Salud con Prevención, de las cuales sólo 25 corresponden al embarazo en la adolescencia, 4 al aborto, 2 a la adopción y 2 a la anticoncepción (13).

\section{Antecedentes y justificación}

La Federación Colombiana de Sociedades de Obstetricia y Ginecología (FECOLSOG) en razón de la importancia que tiene el tema, lo adoptó como punto principal de discusión de la Segunda Reunión del Grupo Bolivariano de FLASOG, conformado por Colombia, Bolivia, Ecuador, Perú y Venezuela y que se realizó en el Centro de Convenciones Gonzalo Jiménez de Quesada los días 1, 2 y 3 de septiembre de 1988.

El objetivo general de dicha reunión fue promover a nivel de las Federaciones o Sociedades Nacionales de los países del Grupo, el estudio de problemas del Embarazo en la Adolescencia y como objetivos específicos, sentar las bases para la realización de proyectos colaborativos multicéntricos de investigación y servicio a las adolescentes en el área de la reproducción, y desencadenar un proceso de atención a la población adolescente de la región en la prevención y manejo del embarazo indeseado.

La Federación Latinoamericana de Sociedades de Obstetricia y Ginecología (FLASOG) entidad que agrupa a las Sociedades y Federaciones Científicas de Ginecología y Obstetricia de todos los países latinoamericanos, representa por lo tanto un potencial de investigación y desarrollo en todos los aspectos de la salud reproductiva, con énfasis en su proyección social, tiene establecidos grupos regionales de acuerdo con la localización geográfica de los países miembros. Entre los objetivos de los grupos figuran los siguientes:

"Organizar jornadas, reuniones o cursos de actualización que permitan el análisis y el conocimiento de los problemas regionales y el estudio de nuevos programas en curso".

"Propiciar programas de investigación multinacional sobre temas de interés común".

Estos grupos tienen la obligación de reunirse cada año y le correspondió a la Federación Colombiana de Sociedades de Obstetricia y Ginecología realizar la segunda reunión antes mencionada.

La reunión tuvo la modalidad de taller con el objeto de darle características de grupo de trabajo, con el propósito de elaborar un proyecto colaborativo multinacional liderado por las Sociedades Federaciones nacionales que diera las bases para un trabajo continuo cuyos resultados tengan real trascendencia en la salud reproductiva del adolescente.

Asistieron a esta reunión delegados de los cinco países del Grupo Bolivariano (Bolivia, Colombia, Ecuador, Perú y Venezuela) y observadores nacionales afines al tema (Minsalud, Asociación Salud con Prevención). 
La propuesta de FECOLSOG de tomar como tema central de la reunión el embarazo en adolescentes y la modalidad de trabajo en forma de taller fue aceptada en forma unánime por todos los delegados.

En la primera sesión se hizo una revisión del tema y se concluyó que era un problema médico-social común a todos los países de la región, cuyas reales dimensiones se intuyen, pero no se tienen debidamente analizadas por carencia de investigaciones suficientemente representantivas; por esta razón se concluyó que el problema ameritaba el diseño de un Estudio Colaborativo multinacional que permitiera allegar datos suficientes para obtener un diagnóstico real, con base en el cual se pueda proponer a las instituciones privadas y gubernamentales, acciones que modifiquen la actual situación.

La Federación Colombiana de Sociedades de Obstetricia y Ginecología (FECOLSOG) diseñó una investigación colaborativa retrospectiva en la cual colaboraron 11 instituciones del país.

\section{Propósitos y objetivos PROPOSITOS}

Analizar la información recolectada en forma retrospectiva del embarazo en la adolescencia en las instituciones colombianas participantes.

\section{OBJETIVO GENERAL}

Determinar en forma consistente y confiable el problema del embarazo en la adolescencia en cada una de las instituciones participantes de los cinco países de la región.

\section{OBJETIVOS ESPECIFICOS}

- Identificar la magnitud del problema.

- Identificar algunos aspectos socio-demográficos del embarazo en la adolescencia.

- Identificar los principales aspectos obstétricos del embarazo en la adolescente.

- Identificar la principal patología de la madre adolescente y su recién nacido.

\section{Material y métodos \\ POBLACION}

Todos los embarazos en adolescentes, que ocurrieron en las instituciones participantes de enero 1o. de 1987 hasta el 31 de diciembre de 1987.

\section{Tabla 1 \\ CASOS POR INSTITUCION}

\begin{tabular}{|c|c|c|c|c|}
\hline INSTITUCION & CODIGO & $\begin{array}{l}\text { TOTAL } \\
\text { PARTOS } \\
\end{array}$ & $\begin{array}{l}\text { NUMERO } \\
\text { ADOLESCENTES } \\
\end{array}$ & PORCETA JE \\
\hline SAN JUAN OE DIOS DE ARMENIA & 1 & 2944 & 421 & 14.30 \\
\hline MATERNO INFANTIL DE BOGOTA & 2 & 8692 & 246 & 2.83 \\
\hline SAN JOSE DE BOGOTA & 3 & 3986 & 156 & 3.91 \\
\hline HOSPITAL GENERAL DE MEDELLIN & 4 & 16777 & 1.222 & 7.28 \\
\hline INSTITUTO DE SEGUROS SOCIALES DE CUCUTA & 5 & 1633 & 53 & 3.24 \\
\hline REGIONAL DE VILLAVICENCIO & 6 & 3860 & 385 & 9.97 \\
\hline SAN IGNAGIO DE BOGOTA & 7 & 3518 & 97 & 2.75 \\
\hline SAN RAFAEL DE FACATATIVA & 8 & 1792 & 196 & 10.93 \\
\hline DEPARTAMENTAL OE NARIÑO & 9 & 2837 & 126 & 4.44 \\
\hline UNIVERSITARIO DE POPAYAN & 10 & 3768 & 129 & 3.42 \\
\hline RAMON GONZALEZ Z VALENCIA DE BUCARAMANGA & 11 & 4690 & 333 & 7.10 \\
\hline \multicolumn{2}{|l|}{ TOTAL } & 54497 & 3.364 & 6.17 \\
\hline
\end{tabular}

\section{Figura 1 \\ DISTRIBUCION POR EDAD}

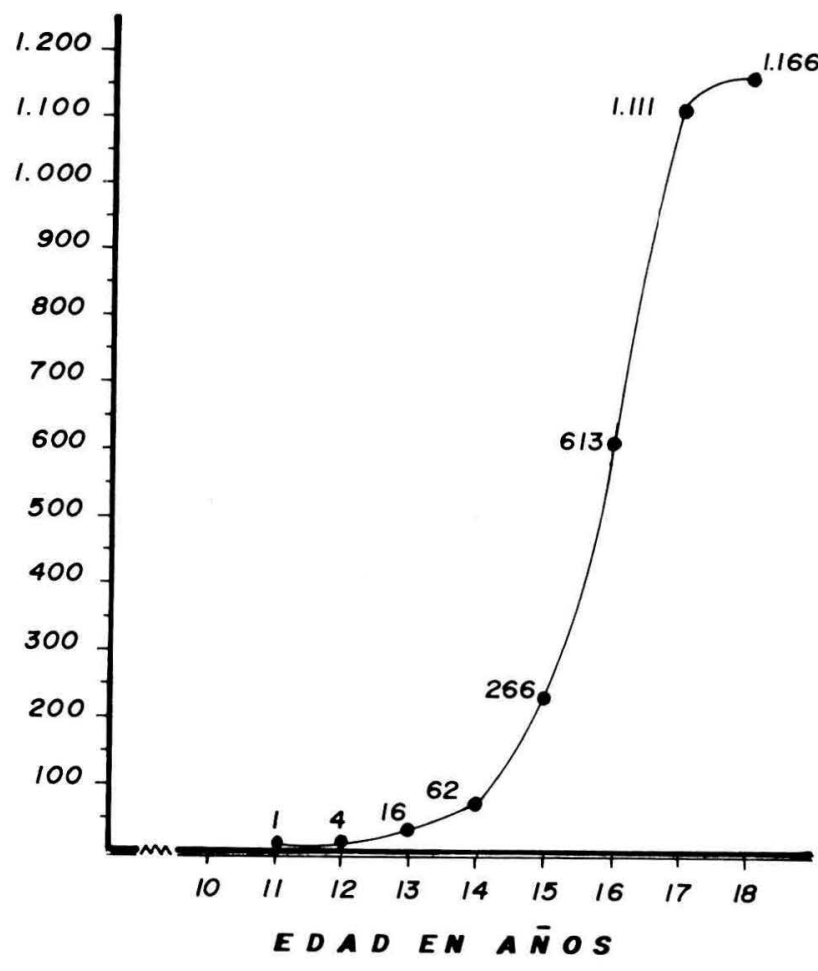

SIN INFORMACION 13.

$\bar{x} \quad 16.9$

\section{RECOLECCION DE LA INFORMACION}

Recolección retrospectiva de las historias clínicas de los embarazos en adolescentes en el período antes señalado, utilizando un formulario precodificado con su respectivo instructivo (Anexos 1, 2 y 3 ).

PROCESAMIENTO DE LA INFORMACION RECOLECTADA

ANALISIS DE LA INFORMACION ELABORACION DEL DOCUMENTO

\section{Resultados}

Participaron 11 instituciones de II y III nivel, reportando todos los casos atendidos de pacientes obstétricas hasta los 18 años, del 1o. de enero al 31 de diciembre de 1987.

Durante este período se atendieron en las instituciones 54.497 pacientes, correspondiendo a menores de 19 años $3.364(6.17 \%)$ de las cuales se descartaron por diversas razones 112 formularios quedando para el análisis 3.252 que constituyen el universo del estudio. La institución con mayor proporción de adolescentes fue el Hospital San Juan de Dios de Armenia con 14.3\% y la institución con menor proporción el Hospital San Ignacio de Bogotá con 2.75\% (Tabla 1).

\section{EDAD MATERNA}

Se encontraron 349 pacientes menores de 16 años (10.77\%) con un aumento de casi el doble de los 15 a los 16 años $(8.21 \%$ - 18.93\%) y entre los 16 y los 17 años (18.93\% - 34.30\%) estabilizándose el incremento en los 17 y 18 años $(34,30 \%$ y $36 \%)$. Figura 1 . 
Figura 2

ESTADO MARITAL

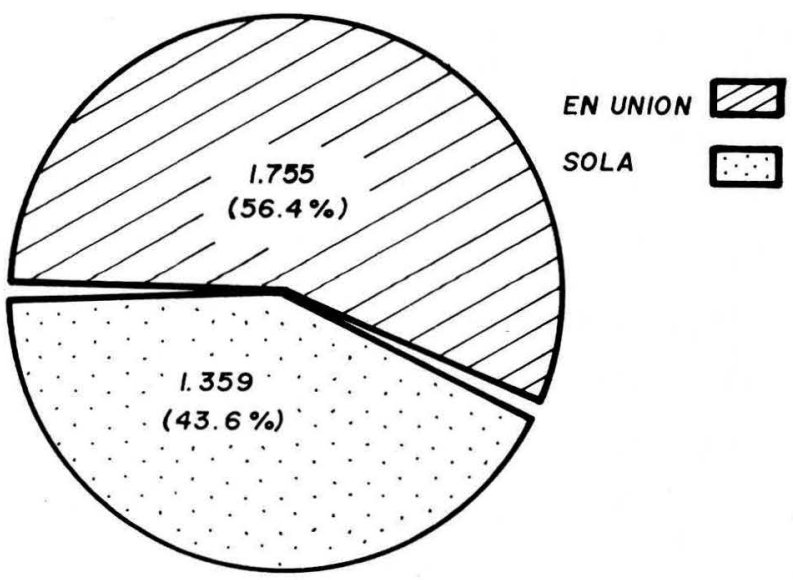

- SIN INFORMACION 138.

\section{ESTADO MARITAL}

Casi la mitad de las madres, $46.6 \%$, se encontraron solas y $56.4 \%$ en unión. Figura 2.

EDAD DE LA MENARQUIA

La menarquia se presentó en su mayoría entre los 12 y 13 años con un promedio de edad de $12.8 \%$. Figura 3.

EMBARAZOS PREVIOS

La gran mayoría de las pacientes, $85 \%$, fueron primigestantes. El $15 \%$ tenía antecedentes de embarazos anteriores. Figura 4.

Figura 3

EDAD DE LA MENARgUIA

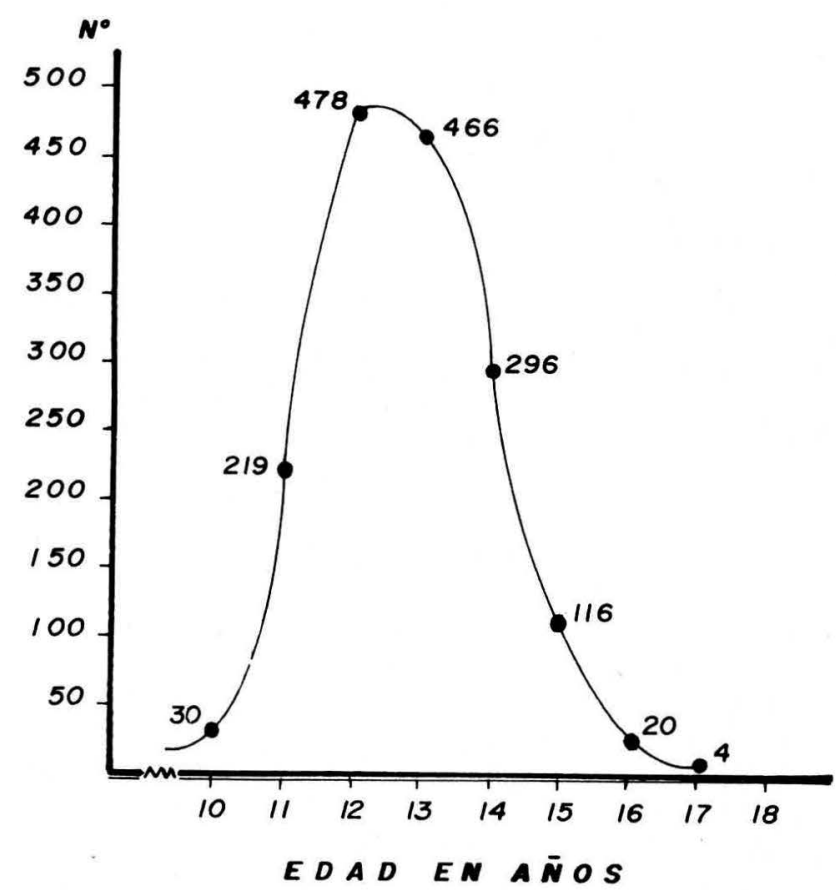

SIN INFORMACION 1.623.
Figura 4

NUMERO DE EMBARAZOS

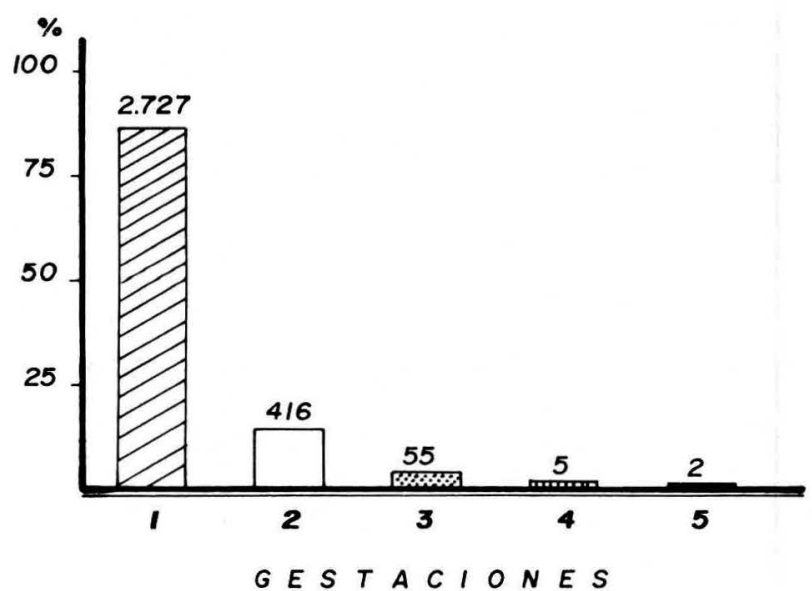

- SIN INFORMACION 47

\section{PARTOS PREVIOS}

La mayoría de las pacientes, $73.7 \%$ fueron nulíparas. Un número considerable de adolescentes, $26.3 \%$, tenían antecedentes de partos anteriores. Figura 5.

ABORTOS PREVIOS

La gran mayoría de pacientes no tenían antecedentes de aborto, $96.5 \%$. Solamente 114 pacientes, $3.5 \%$, tenían antecedentes de 1 o más abortos. Figura 6.

CONTROL PRENATAL

Más de la mitad de las pacientes no tuvieron ningún control prenatal, $58.2 \%$. Figura 7 .

NUMERO DE CONTROLES PRENATALES

De las pacientes con control prenatal una cuarta parte de ellas, $25.7 \%$, solamente tuvieron de 1 a 2 controles. El $63.9 \%$ tuvo de 3 a 6 controles y más de 6 controles el $10.4 \%$. El promedio de controles prenatales fue de $4.01 \%$. Figura 8.

MORBILIDAD DEL EMBARAZO

Más de la cuarta parte de los embarazos tuvieron algún tipo de patología, 28.7\%. Figura 9.

\section{Figura 5}

\section{PARIDAD}

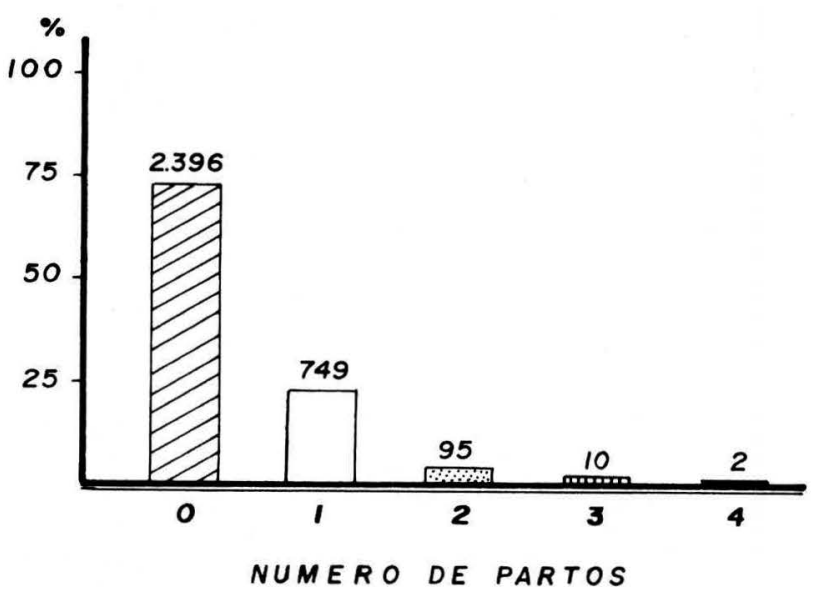


TIPO DE MORBILIDAD ASOCIADA AL EMBARAZO

Las tres primeras patologías asociadas fueron: la ruptura prematura de membranas $28.4 \%$, infecciones $18.9 \%$, y la hipertensión asociada al embarazo $18.1 \%$. Se presentaron 7 muertes maternas antes del parto $0.8 \%$. Figura 10 .

De las infecciones las más frecuentes fueron la infección urinaria $32.3 \%$ y la cervicitis y vaginitis $24.9 \%$, y las enfermedades venéreas $21.1 \%$. Si consideramos la cervicitis y vaginitis y las enfermedades venéreas como enfermedades sexualmente transmisibles, casi la mitad de las pacientes con infección tuvieron este tipo de patología, 46\%. Figura $10 \mathrm{~A}$.

De las hipertensiones asociadas al embarazo la principal fue la preeclampsia leve con $67 \%$, siguiendo en frecuencia la preeclampsia grave con $10.4 \%$ y la eclampsia con $9.1 \%$. Es decir que la toxemia constituyó el $86.5 \%$ de la hipertensión asociada al embarazo. Figura 10B.

Figura 6 ABORTOS

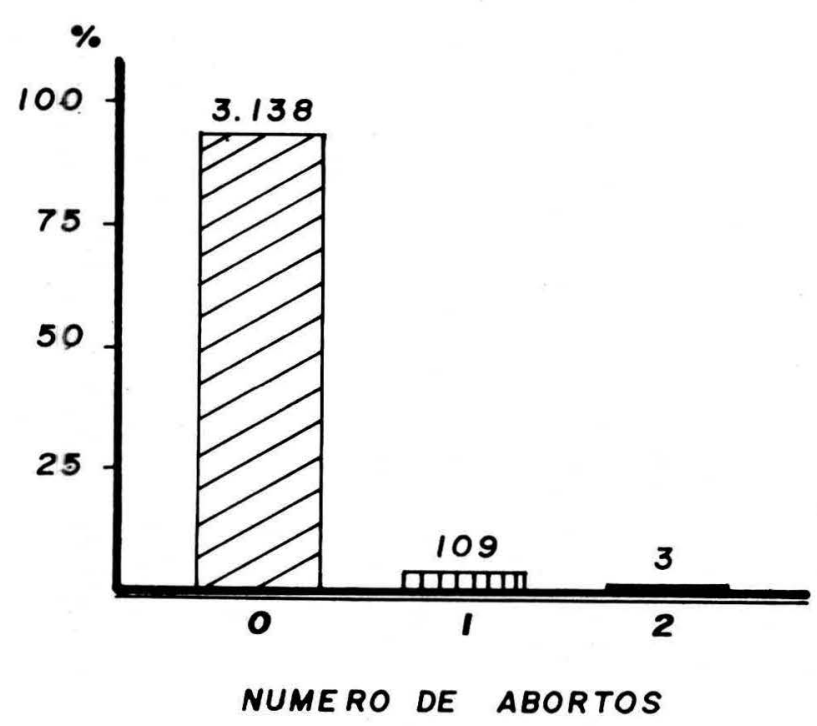

Figura 7 CONTROL PRENATAL

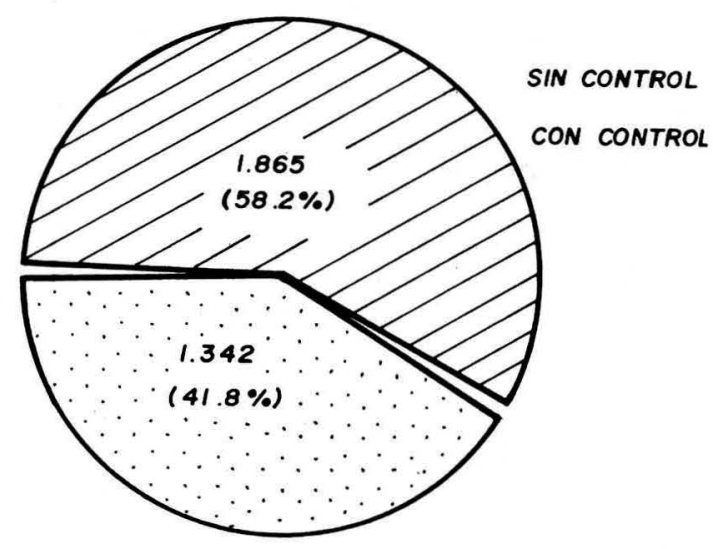

- SIN INFORMACION 45.
Figura 8

NUMERO DE CONTROLES PRENATALES

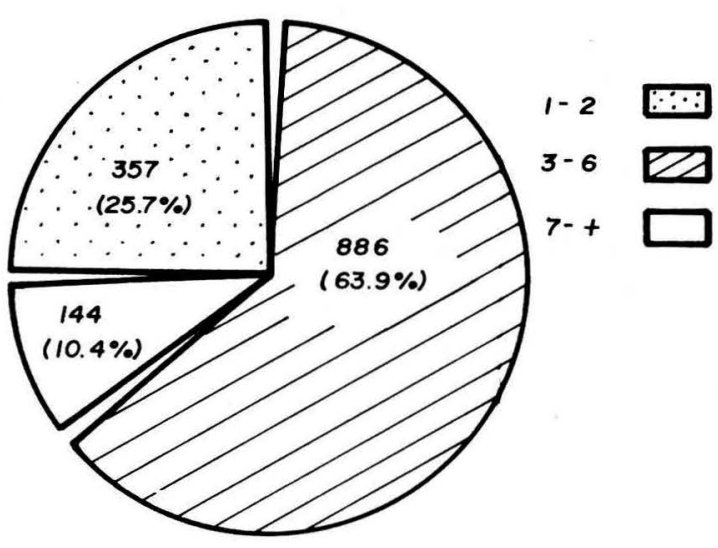

- SIN CONTROL $1.865 . \quad \bar{x} 4.01$

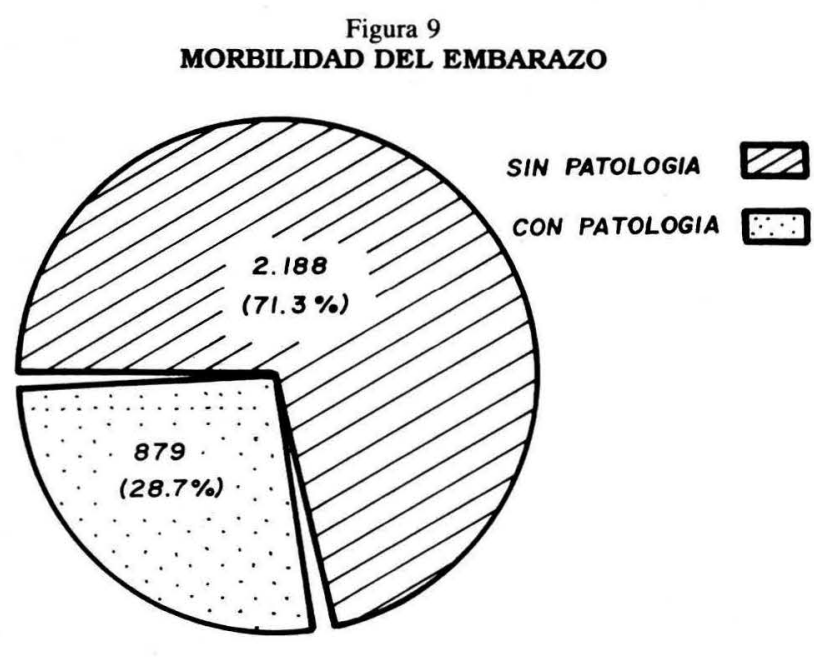

- SIN INFORMACION 185.

\section{EDAD GESTACIONAL}

La gran mayoría de los embarazos fueron a término, $76.4 \%$, siendo los prematuros casi una cuarta parte, $21.5 \%$. Figura 11.

TIPO DE ATENCION DEL PARTO

La mayoría de los partos fueron espontáneos, 78.3\%. Casi una cuarta parte fueron intervenidos, 21.7\%. Figura 12. TIPO DE INTERVENCION

La principal intervención fue la cesárea con $74.9 \%$ siguiendo en frecuencia el fórceps o las espátulas con $20.90 \%$. Figura 13.

\section{COMPLICACION DEL PARTO}

La gran mayoría de los partos fueron no complicados, $82.6 \%$. Con alguna complicación $17.4 \%$. Figura 14. TIPO DE COMPLICACION DEL PARTO

La principal complicación fue la desproporción cefalopélica, $29.3 \%$, siguiendo la distocia de presentación, $18.7 \%$, 
Figura 10

TIPO DE MORBILIDAD ASOCIADA AL EMBARAZO

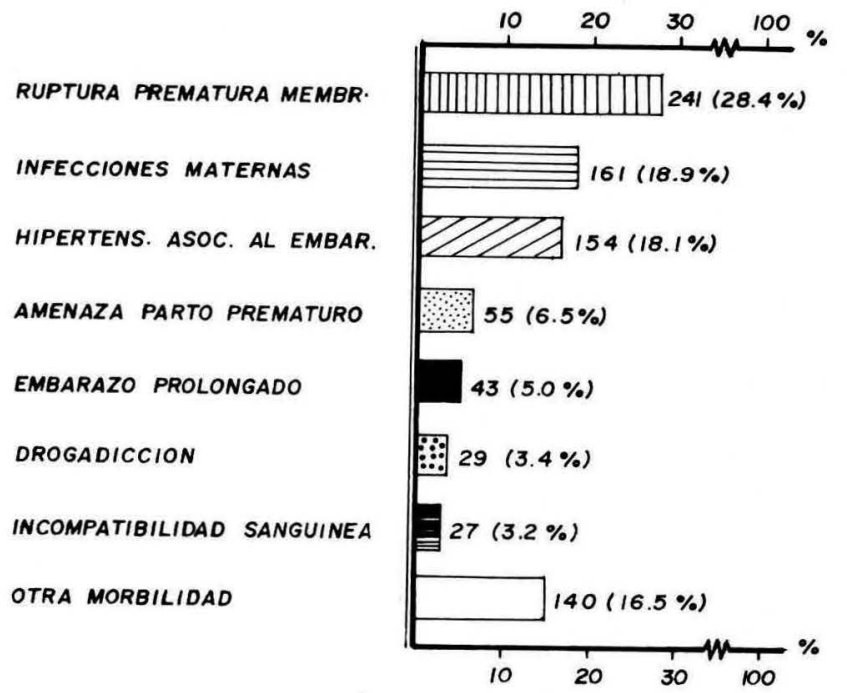

- SIN INFORMACION 29.

y el trabajo de parto prolongado, 9.1\%. Figura 15.

PESO DEL RECIEN NACIDO

La gran mayoría fueron de pesos normales, $83.6 \%$. De bajo peso $9.7 \%$ y macrosómico $5.3 \%$. El promedio de peso fue de 2.970 gramos. Figura 16.

APGAR

La calificación de apgar al minuto fue en su gran mayoría de 7 y más $89.9 \%$. El $10.1 \%$ tuvo apgar al minuto por debajo de 7. Figura 17A.

En la calificación de apgar a los cinco minutos la mayoría 96.5\% tuvieron apgar de 7 o más y solamente $3.5 \%$ fueron calificados con menos de 7. Figura 17B.

PATOLOGIA DEL RECIEN NACIDO

La gran mayoría de los recién nacidos no tuvieron ninguna patología, $86.9 \%$. El $13.1 \%$ tuvo algún tipo de patología. Figura 18.

Figura 10-A

INFECCIONES MATERNAS

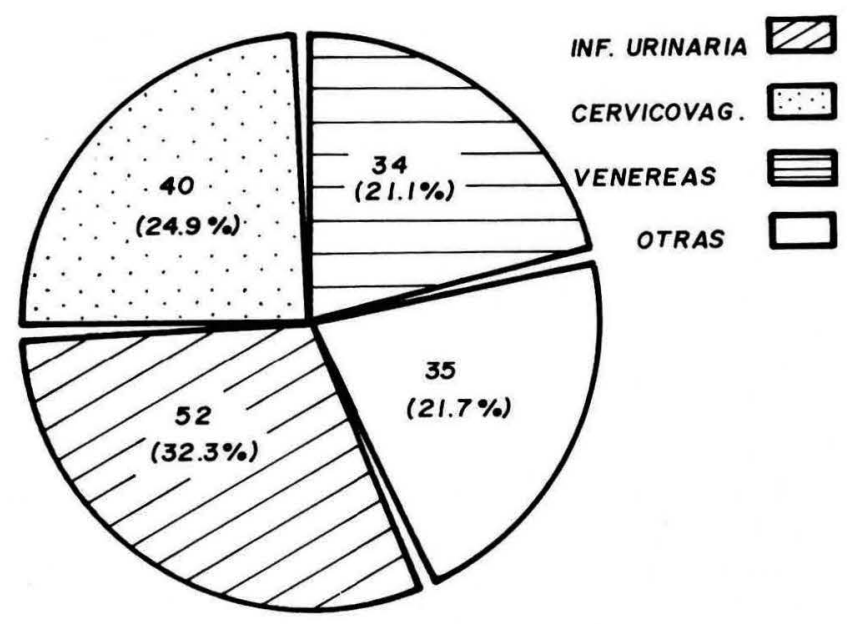

Figura 10-B

HIPERTENSION ASOCLADA AL EMBARAZO

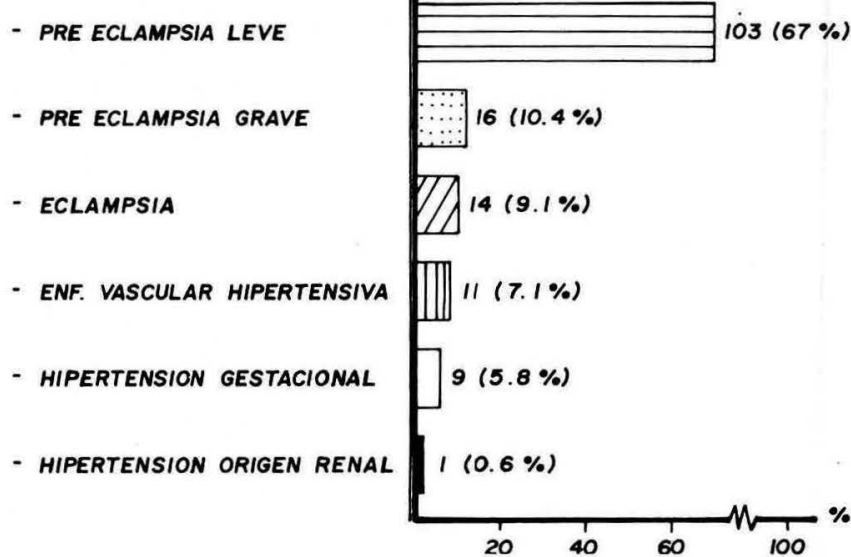

TIPO DE PATOLOGIA DEL RECIEN NACIDO

La principal patología del recién nacido fue la prematurez, $50.2 \%$, siguiendo las anomalías congénitas con $8.1 \%$, el síndrome de dificultad respiratoria $7.4 \%$, y el recién nacido deprimido $7.1 \%$. Figura 19.

COMPLICACION DEL PUERPERIO

La gran mayoría de las pacientes no presentaron ningún tipo de patología en el puerperio, $95.5 \%$. Sólo el $4.5 \%$ presentó algún tipo de patología. Figura 20.

TIPO DE COMPLICACION DEL PUERPERIO

La principal patología encontrada fue la endometritis, $67 \%$, siguiendo la hemorragia con $13 \%$ y otras infecciones 9.6\% . La infección constituyó el $76.6 \%$ de las complicaciones del puerperio. En este período se presentaron 2 muertes maternas, $1.7 \%$. Figura 21.

\section{ESTANCIA HOSPITALARIA}

Casi la mitad de las pacientes sólo permanecieron un día en el hospital, $46.7 \%$. Dos días $31.9 \%$ y tres o más días $21.4 \%$. Figura 22.

Figura 11

TERMINO DEL EMBARAZO POR EDAD GESTACIONAL
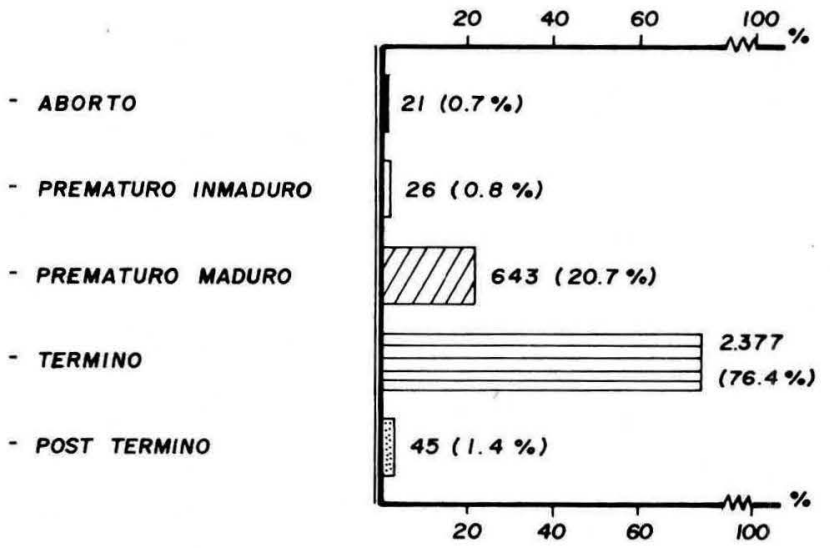

- SIN INFORMACION 140.

$\bar{x} 38$ 
Tabla 2

MORBILIDAD ASOCIADA AL EMBARAZO

SEGUN EDAD MATERNA EN PORCENTAJE

\begin{tabular}{|c|c|c|c|c|}
\hline \multirow{2}{*}{ MORBILIDAD } & \multicolumn{4}{|c|}{ EDAD MATERNA EN PORCENTAJE } \\
\hline & 15 años & 16 oños & 17 onos & 18 años \\
\hline RP M & 23.3 & 28.1 & 22.3 & 36.0 \\
\hline INFECCIONES & 18.6 & 20.3 & 19.5 & 17.8 \\
\hline MIPERTENSION & 25.6 & 18.5 & 17.8 & 16.0 \\
\hline$A P P$ & 3.5 & 7.3 & 6.7 & 6.6 \\
\hline EMBARAZO PROLONGADO & 5.8 & 1.7 & 7.1 & 4.9 \\
\hline DROGADICCION & 1.2 & 2.2 & 3.7 & 4.5 \\
\hline INCOMPATIBILIDAD SANGUINEA & 3.5 & 3.4 & 4.4 & 1.7 \\
\hline OTRA & 18.5 & 18.5 & 18.5 & 12.5 \\
\hline TOTAL & 100 & 100 & 100 & 100 \\
\hline
\end{tabular}

TIPO DE MORBILIDAD ASOCIADA AL EMBARAZO SEGUN EDAD MATERNA

En pacientes de 15 años la primera morbilidad fue la hipertensión asociada al embarazo con $25.6 \%$ y en el resto de edades la primera causa fue la ruptura prematura de membranas, $28.1 \%$ en los 16 años, $22.3 \%$ en los 17 años, y $36.0 \%$ en los 18 años. La infección ocupó el tercer lugar en las pacientes de 15 años con $18.6 \%$ y el segundo lugar en el resto de edades: $20.3 \%$ en los 16 años, $19.5 \%$ en los 17 años, y $17.8 \%$ en los 18 años. Tabla 2 .

Tabla 3

COMPLICACIONES DEL PUERPERIO SEGUN EDAD MATERNA EN PORCENTAJE

\begin{tabular}{|c|c|c|c|c|}
\hline \multirow{2}{*}{ COMPLICACION } & \multicolumn{4}{|c|}{ EDAD EN PORCENTAJE } \\
\hline & 15 ohios & 16 oños & 17 años & 18 onos \\
\hline ENDOMETRITIS & 60.0 & 71.0 & 57.9 & 44.4 \\
\hline HEMORRAGIA & 10.0 & 9.7 & 5.3 & 25.0 \\
\hline OTRAS INFECCIONES & -- & 6.5 & 10.5 & 13.9 \\
\hline MUERTE MATERNA & 10.0 & 3.1 & -- & -- \\
\hline OTRAS COMPLICACIONES & 20.0 & 9.7 & 26.3 & 16.7 \\
\hline TOTAL & 100 & 100 & 100 & 100 \\
\hline
\end{tabular}

Tabla 4

MORBILIDAD DEL EMBARAZO SEGUN CONTROL PRENATAL EN PORCENTAJE

\begin{tabular}{|l|c|c|c|}
\hline \multirow{2}{*}{ MORBILIDAD.— } & \multicolumn{2}{|c|}{ CONTROL } & \multirow{2}{*}{ TOTAL } \\
\cline { 2 - 4 } & $S I$ & NO & \\
\hline INFECCION & 48.3 & 63.9 & 100 \\
HIPERTENSION & 31.1 & 68.9 & 100 \\
A P P & 32.9 & 67.1 & 100 \\
EMBARAZO PROLONGADO & 47.2 & 52.8 & 100 \\
DROGADICCION & 30.2 & 69.8 & 100 \\
INCOMPATIBILIOAO SANGUINEA & 48.3 & 51.7 & 100 \\
OTRA & 14.8 & 85.2 & 100 \\
\hline
\end{tabular}

Tabla 5

TIPO DE INTERVENCION SEGUN

CONTROL PRENATAL EN PORCENTAJE

\begin{tabular}{|l|c|c|c|}
\hline \multirow{2}{*}{ INTERVENCION } & \multicolumn{2}{|c|}{ CONTROL } & \multirow{2}{*}{ TOTAL } \\
\cline { 2 - 3 } & SI & N O & 100 \\
\hline CESAREA & 34.8 & 65.2 & 100 \\
FORCEPS & 31.9 & 68.1 & 100 \\
OTRA & 31.0 & 69.0 & \\
\hline
\end{tabular}

Tabla 6

TIPO DE COMPLICACION DEL PARTO

SEGUN CONTROL PRENATAL EN PORCENTAJE

\begin{tabular}{|l|c|c|c|}
\hline \multirow{2}{*}{ COMPLICACION } & \multicolumn{2}{|c|}{ CONTROL } & \multirow{2}{*}{ TOTAL } \\
\cline { 2 - 3 } & SI & NO & \\
\hline D C P & 38.7 & 61.3 & 100 \\
OISTOCIA DE PRESENTACION & 46.7 & 53.3 & 100 \\
T P P & 41.9 & 58.1 & 100 \\
S F A & 27.5 & 72.5 & 100 \\
HEMORRAGIA & 35.3 & 64.7 & 100 \\
COMPRESION DEL CORDON & 22.6 & 77.4 & 100 \\
OTRA & 52.1 & 47.9 & 100 \\
\hline
\end{tabular}

\section{TIPO DE COMPLICACIONES DEL PUERPERIO SEGUN} EDAD MATERNA

La primera morbilidad del puerperio en todas las edades fue la endometritis: $60.0 \%$ a los 15 años, $71.0 \%$ a los 16 años, $57.9 \%$ a los 17 años, y $44.4 \%$ a los 18 años. La hemorragia ocupó el segundo lugar en todas las edades a excepción de los 17 años que ocupó el tercer lugar: 10.0\% en los 15 años, $9.7 \%$ en los 16 años, 5.3\% en los 17 años, y $25.0 \%$ en los 18 años. Las únicas dos muertes puerperales ocurrieron en una paciente de 15 años y en otra paciente de 16 años. Tabla 3.

TIPO DE MORBILIDAD DEL EMBARAZO SEGUN CONTROL PRENATAL

En todas las patologías el porcentaje de morbilidad fue mayor en las pacientes sin control prenatal siendo esta diferencia máyor en la incompatibilidad sanguínea $85.2 \%$, embarazo prolongado $69.8 \%$, infección $68.9 \%$ e hipertensión 67.1\%. Tabla 4 .

Tabla 7

TIPO DE COMPLICACION DEL PUERPERIO SEGUN CONTROL PRENATAL EN PORCENTAJE

\begin{tabular}{|l|c|c|c|}
\hline \multirow{2}{*}{ COMPLICACION } & \multicolumn{2}{c|}{ CONTROL } & \multirow{2}{*}{ TOTAL } \\
\cline { 2 - 4 } & SI & NO & \\
\hline ENDOMETRITIS & 40.0 & 60.0 & 100.0 \\
HEMORRAGIA & 40.0 & 60.0 & 100.0 \\
OTRAS INFECCIONES & 36.4 & 63.6 & 100.0 \\
MUERTE MATERNA & - & 100.0 & 100.0 \\
OTRAS & 61.9 & 38.1 & 100.0 \\
\hline
\end{tabular}


TIPO DE HIPERTENSION SEGUN CONTROL PRENATAL

El porcentaje de parto intervenido fue mayor siempre en las pacientes sin control prenatal $65.2 \%$, en cesárea $68.1 \%$, en fórceps y otro tipo de intervención $69.0 \%$. Tabla 5. TIPO DE COMPLICACION DEL PARTO SEGUN CONTROL PRENATAL

El porcentaje de complicación del parto fue más frecuente en todos los casos en las pacientes sin control prenatal siendo esta diferencia mayor en la compresión del cordón $77.4 \%$, sufrimiento fetal agudo $77.5 \%$, y hemorragia $64.7 \%$. Tabla 6.

TIPO DE COMPLICACION DEL PUERPERIO SEGUN CONTROL PRENATAL

La complicación del puerperio más frecuente fue la infección tanto en pacientes con control prenatal $61.3 \%$, como en las pacientes sin control prenatal $70.8 \%$. El porcentaje de complicaciones fue mayor en las pacientes sin control prenatal $60.0 \%$ en endometritis, $60.0 \%$ en hemorragia, $63.6 \%$ en otras infecciones; y las únicas 2 muertes maternas del puerperio fueron en pacientes sin control prenatal. Tabla 7.

TIPO DE INTERVENCION SEGUN MORBILIDAD ASOCIADA AL EMBARAZO

En las pacientes intervenidas con cesárea las tres primeras patologías asociadas al embarazo fueron en su orden: ruptura prematura de membranas $25.3 \%$, hipertensión $23.7 \%$, y distocia $14.3 \%$. En las pacientes intervenidas con fórceps las 3 primeras patologías asociadas fueron en su orden: ruptura prematura de membranas $29.8 \%$, hipertensión $27.0 \%$ e infección $21.6 \%$. Tabla 8.

PESO EN GRAMOS DEL RECIEN NACIDO SEGUN MORBILIDAD ASOCIADA AL EMBARAZO

La morbilidad asociada al embarazo que tuvo un porcentaje menor de recién nacidos de peso normal fue la amenaza de parto pretérmino con $55.5 \%$. Las entidades que más se asociaron a recién nacidos de peșo menor de 2.500 gramos fueron la amenaza de parto pretérmino con $42.6 \%$, la hipoxia intraparto con $25.0 \%$, y la drogadicción con $20.7 \%$. Tabla 9. ESTANCIA HOSPITALARIA EN DIAS SEGUN MORBILIDAD ASOCIADA AL EMBARAZO

Las tres patologías con estancia hospitalaria de más de dos días fueron en su orden: la distocia con $76.2 \%$, hipertensión $47.0 \%$ y amenaza de parto prematuro $44.5 \%$. Tabla 10.

TIPO DE COMPLICACION DEL PUERPERIO SEGUN TIPO DE INTERVENCION

El porcentaje de complicación del puerperio fue siempre mayor en las pacientes de cesárea que en cualquier otro tipo de intervención. $96.8 \%$ en endometritis, $66.7 \%$ en hemorra-

Tabla 8

TIPO DE INTERVENCION SEGUN MORBILIDAD ASOCIADA AL EMBARAZO EN PORCENTAJE

\begin{tabular}{|c|c|c|c|c|c|c|c|c|c|}
\hline \multirow[b]{2}{*}{ INTERVENCION } & \multicolumn{8}{|c|}{ NORBILIDAO } & \multirow[b]{2}{*}{ TOTAL } \\
\hline & RPA & INFECCION- & - MIPERTENSION & DISTOCIA & $\begin{array}{c}\text { EMBAAAZO } \\
\text { PROLONGADO }\end{array}$ & $A P P$ & $\begin{array}{l}\text { MIPOXIA } \\
\text { INTRAPARTO }\end{array}$ & OTRA & \\
\hline CESAREA & 25.3 & 13.0 & 23.7 & 14.3 & 4.9 & 3.3 & 2.9 & 12.6 & 100 \\
\hline FORCEPS & 29.8 & 21.6 & 27.0 & -- & -- & 5.4 & 3.4 & 10.8 & 100 \\
\hline OTRA & -- & 9.1 & 18.2 & 36.3 & 27.3 & 9.1 & -- & -- & 100 \\
\hline
\end{tabular}

Tabla 9

PESO EN GRAMOS DEL RECIEN NACIDO SEGUN MORBILIDAD ASOCIADA AL EMBARAZO EN PORCENTAJE

\begin{tabular}{|c|c|c|c|c|c|c|c|c|}
\hline \multirow{2}{*}{ PESO EN GRAMOS } & \multicolumn{8}{|c|}{ MORBILIOAD. } \\
\hline & DROGADICCION & HIPERTENSION & INFECCION & $A P P$ & $\begin{array}{l}\text { ENBAAAZO } \\
\text { PROLONGADC }\end{array}$ & $\begin{array}{l}\text { MIPOXIA } \\
\text { INTRAPARTO }\end{array}$ & RP & OTRAS \\
\hline$<-1.500$ & 6.9 & 0.7 & 1.8 & 9.3 & -- & 12.5 & 2.5 & 1.5 \\
\hline $1.300-2.499$ & 13.8 & 17.9 & 8.2 & 33.3 & 4.9 & 12.5 & 13.4 & 8.1 \\
\hline $2.800-3.799$ & 75.9 & 76.6 & B5.2 & 55.5 & 85.3 & 68.7 & 78.2 & 65.3 \\
\hline$\geq-3.000$ & 3.4 & 4.8 & 4.8 & 1.8 & 9.8 & 63 & 5.9 & 5.1 \\
\hline TOTAL & 100 & 100 & 100 & 100 & 100 & 100 & 100 & 100 \\
\hline
\end{tabular}

Tabla 10

ESTANCIA HOSPITALARIA EN DIAS SEGUN MORBILIDAD ASOCIADA AL EMBARAZO EN PORCENTAJE

\begin{tabular}{|c|c|c|c|c|c|c|c|c|}
\hline \multirow{2}{*}{ ESTANCIA EN DIAS } & \multicolumn{8}{|c|}{ MORBILIOAO } \\
\hline & DISTOCIA & HIPERTENSIOM & INFECGION & $A P P$ & $\begin{array}{r}\text { EMBARAZO } \\
\text { PROLONGADO }\end{array}$ & $\begin{array}{l}\text { HIPOXIA } \\
\text { INTRAPARTO }\end{array}$ & RP W & OTRAS \\
\hline uno & 9.5 & 24.5 & 29.4 & 23.9 & 37.2 & 3.9 & 43.1 & 26.2 \\
\hline oos & 14.3 & 28.5 & 36.6 & 29.6 & 30.2 & 29.4 & 32.2 & 27.6 \\
\hline $3-5$ & 64.3 & 35.1 & 28.8 & 37.1 & 27.9 & 64.7 & 19.7 & 44.0 \\
\hline 6 Y WAS & 11.9 & 11.9 & 3.2 & 7.4 & 4.7 & -- & 5.0 & 2.2 \\
\hline TOTAL & 100 & 100 & 100 & 100 & 100 & 100 & 100 & 100 \\
\hline
\end{tabular}

Tabla 11

COMPLICACIONES DEL PUERPERIO SEGUN TIPO DE INTERVENCION EN PORCENTAJE

\begin{tabular}{|l|c|c|c|c|}
\hline \multirow{2}{*}{ COMPLICACION } & \multicolumn{3}{|c|}{ INTERVENCION } & \multirow{2}{*}{ TOTAL } \\
\cline { 2 - 5 } & CESAREA & FORCEPS & OTRA & \\
\hline ENDOMETRITIS & 96.8 & 3.2 & -- & 100 \\
HEMORRAGIA & 66.7 & 33.3 & -- & 100 \\
OTRAS INFECCIONES & 100 & -- & -- & 100 \\
MUERTE MATERNA & -- & -- & 100 & 100 \\
OTRAS & 60.0 & 40.0 & -- & 100 \\
\hline
\end{tabular}

gia, todos los casos de otras infecciones y $60.0 \%$ de otras complicaciones. Tabla 11.

ESTANCIA HOSPITALARIA EN DIAS SEGUN TIPO DE INTERVENCION

El procedimiento con mayor estancia hospitalaria fue la cesárea: con más de dos días $64.4 \%$; y el de menor estancia hospitalaria fue el fórceps, $69.1 \%$, con menos de 3 días. Tabla 12.

APGAR AL MINUTO SEGUN TIPO DE COMPLICACION DEL PARTO

Las complicaciones con mayor porcentaje de apgar por debajo de 7 al minuto fueron en su orden: el sufrimiento fetal agudo $35.9 \%$, trabajo de parto prolongado $28.5 \%$, y presentación distócica $26.4 \%$. Tabla 13.

APGAR A LOS CINCO MINUTOS SEGUN TIPO DE COMPLICACION DEL PARTO

Dentro de los apgar menores de 7 a los 5 minutos las complicaciones del parto con mayor proporción fueron en su orden: compresión del cordón $12.9 \%$, trabajo de parto prolongado $9.5 \%$ y desproporción cefalopélvica $7.8 \%$. Las complicaciones del parto con mayor porcentaje de apgar por encima de 7 a los 5 minutos fueron en su orden: el sufrimiento 
Tabla 12

ESTANCIA HOSPITALARIA EN DIAS SEGUN TIPO DE INTERVENCION

\begin{tabular}{|l|c|c|c|}
\hline \multirow{2}{*}{ ESTANCIA } & \multicolumn{3}{|c|}{ INTERVENCION. } \\
\cline { 2 - 4 } & CESAREA & FORCEPS & OTRA \\
\hline UNO & 6.4 & 27.5 & 17.2 \\
DOS & 29.2 & 41.6 & 27.6 \\
$3-3$ & 51.3 & 23.2 & 51.8 \\
6 Y MAS & 13.1 & 7.7 & 3.4 \\
\hline TOTAL & 100 & 100 & 100 \\
\hline
\end{tabular}

Tabla 13

APGAR AL MINUTO SEGUN TIPO DE COMPLICACION DEL PARTO EN PORCENTAJE

\begin{tabular}{|c|c|c|c|c|c|c|c|}
\hline \multirow{2}{*}{ APGAR Ne mINUTO } & \multicolumn{7}{|c|}{ TIPO DE COMPLICACION OEL PARTO } \\
\hline & OCP & $\begin{array}{l}\text { PRESENTACION } \\
\text { OISTOCICA }\end{array}$ & $T P P$ & HEMORRAGU & $\begin{array}{l}\text { COMPAESION } \\
\text { DEL COROON }\end{array}$ & SFA & OTRA \\
\hline $0-3$ & 0.6 & 3.5 & 7.1 & 0.0 & 3.2 & 6.1 & 17.0 \\
\hline $4-6$ & 12.1 & 20.8 & 21.4 & .. & 16.1 & 30.0 & 13.7 \\
\hline I Y was & res & 73.6 & 71.4 & 02.4 & 00.7 & C. I I & 69.3 \\
\hline TOTAL & 100 & 100 & 100 & 100 & 100 & 100 & 100 \\
\hline
\end{tabular}

Tabla 14

APGAR A LO CINCO MINUTOS SEGUN TIPO DE COMPLICACION DEL PARTO EN PORCENTAJE

\begin{tabular}{|c|c|c|c|c|c|c|c|}
\hline \multirow{2}{*}{$\begin{array}{c}\text { APGRR A LOS } \\
\text { CINCO AlNUTOS } \\
.\end{array}$} & \multicolumn{7}{|c|}{ TIPO OE COMPLICACION DEL PARTO } \\
\hline & OCP & $\begin{array}{l}\text { peesentaciom } \\
\text { DISTOCICA }\end{array}$ & TPP & HEMORRAGIA & $\begin{array}{l}\text { COMPRESION } \\
\text { OEL CORDON }\end{array}$ & SFA & orra \\
\hline $0-3$ & 0.7 & 2.2 & 7.1 & 9.1 & 3.2 & 2.6 & 111.4 \\
\hline 4 & 2.1 & 4.4 & 2.4 & -- & 0.7 & -- & 0.1 \\
\hline tr uns & 92.2 & 93.4 & 90.5 & 90.8 & 07.1 & 97.4 & $\bullet 0.5$ \\
\hline TOTAL & 100 & 100 & 100 & 100 & 100 & 100 & 100 \\
\hline
\end{tabular}

Tabla 15

APGAR AL MINUTO SEGUN PESO

EN GRAMOS DEL RECIEN NACIDO EN PORCENTAJE

\begin{tabular}{|c|c|c|c|c|}
\hline \multirow{2}{*}{ APGAR } & \multicolumn{4}{|c|}{ PESO EN GRAMOS } \\
\cline { 2 - 6 } & $<1.500$ & $\begin{array}{l}1.500- \\
2.499\end{array}$ & $\begin{array}{c}2.500- \\
3.799\end{array}$ & 53.800 \\
\hline $0-3$ & 65.9 & 8.3 & 1.2 & -- \\
$4-6$ & 13.6 & 15.3 & 5.8 & 6.6 \\
7 Y MAS & 20.5 & 76.4 & 93.0 & 93.4 \\
\hline TOTAL & 100 & 100 & 100 & 100 \\
\hline
\end{tabular}

fetal agudo $97.4 \%$, distocia de presentación $93.4 \%$ y desproporción cefalopélvica $92.2 \%$. Tabla 14 .

APGAR AL MINUTO SEGUN PESO EN GRAMOS DEL RECIEN NACIDO

La gran mayoría de recién nacidos con peso menor de 1.500 gramos tuvieron apgar al minuto menor de siete, $79.5 \%$. En recién nacidos con pesos entre 1.500 y 2.499 gramos, casi la cuarta parte tuvieron apgar menor de siete, 23.6\%. En recién nacidos con pesos entre 2.500 y 3.799 gramos sólo el $7.0 \%$ tuvieron apgar por debajo de 7 y en los recién nacidos de más de 3.800 gramos sólo el $6.6 \%$ tuvieron apgar por debajo de 7. Tabla 15.

APGAR A LOS CINCO MINUTOS SEGUN PESO EN GRAMOS DEL RECIEN NACIDO

La mayoría de los recién nacidos con peso menor de 1.500 gramos tuvieron apgar a los cinco minutos menor de

Tabla 16

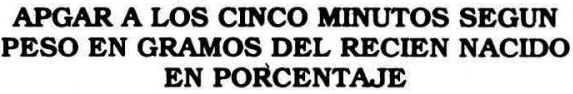

\begin{tabular}{|c|c|c|c|c|}
\hline \multirow{2}{*}{ APGAR } & \multicolumn{4}{|c|}{ PESO EN GRAMOS } \\
\cline { 2 - 6 } & $<1500$ & $\begin{array}{l}1 \\
2.490\end{array}$ & $\begin{array}{l}2 \\
2.790-\end{array}$ & $>3800$ \\
\hline $0-3$ & 45.5 & 5.9 & 0.6 & - \\
$4-6$ & 18.2 & 3.6 & 0.8 & 1.2 \\
\hline Y MAS & 36.3 & 90.5 & 98.6 & 98.8 \\
\hline TOTAL & 100 & 100 & 100 & 100 \\
\hline
\end{tabular}

Tabla 17

COMPLICACION DEL RECIEN NACIDO SEGUN APGAR AL MINUTO EN PORCENTANE

\begin{tabular}{|l|c|c|c|c|}
\hline \multirow{2}{*}{ COMPLICACION } & \multicolumn{3}{|c|}{ APGAR AL MINUTO } & \multirow{2}{*}{ TOTAL } \\
\cline { 2 - 4 } & $0-3$ & $4-6$ & 7 Y MAS & \\
\hline PAEMATUREZ & 15.8 & 16.3 & 67.9 & 100 \\
ANOMALIA CONGENITA & 12.1 & 24.2 & 63.6 & 100 \\
S D N & 24.1 & 55.2 & 20.7 & 100 \\
RECIEN NAGIDO DEPRIMIDO & 7.7 & 34.6 & 57.7 & 100 \\
HEMORRAGIA & 8.0 & 6.0 & 84.0 & 100 \\
MAGROSOMIA & 18.2 & 4.5 & 77.3 & 100 \\
POST-MADURO & -- & 27.8 & 72.2 & 100 \\
INFECGION & 13.3 & 6.7 & 80.0 & 100 \\
OTRA & 45.1 & 11.5 & 43.4 & 100 \\
\hline
\end{tabular}

Tabla 18

COMPLICACIONES DEL RECIEN NACIDO SEGUN APGAR A LOS CINCO MINUTOS EN PORCENTAJE

\begin{tabular}{|l|c|c|c|c|}
\hline \multirow{2}{*}{ COMPLICACION } & \multicolumn{2}{|c|}{ APGAR A LOS CINCO MINUTOS } & \multirow{2}{*}{ TOTAL } \\
\cline { 2 - 4 } & $0-3$ & $4-6$ & 7 Y MAS & \\
\hline PREMATUREZ & 10.8 & 5.9 & 83.3 & 100 \\
ANOMALIAS CONGENITAS & 9.1 & 3.0 & 87.9 & 100 \\
S D R & 20.7 & 34.5 & 44.8 & 100 \\
RN DEPRIMIDO & 7.7 & 3.8 & 88.5 & 100 \\
HEMORRAGIA & 4.0 & -- & 96.0 & 100 \\
MACROSOMIA & 13.6 & 4.5 & 81.9 & 100 \\
POST - MADURO & -- & 5.9 & 94.1 & 100 \\
INFECCION & 6.7 & 13.3 & 80.1 & 100 \\
OTRA & 45.1 & 2.0 & 52.9 & 100 \\
\hline
\end{tabular}


Tabla 19

ESTANCIA HOSPITALARIA EN DIAS SEGUN COMPLICACION DEL PUERPERIO

\begin{tabular}{|l|c|c|c|c|c|}
\hline \multirow{2}{*}{ ESTANCIA } & \multicolumn{5}{|c|}{ COMPLICACION } \\
\cline { 2 - 6 } & ENOOMETRITIS & MEMORRAGIA & $\begin{array}{c}\text { OTRAS } \\
\text { INFEGCIONES }\end{array}$ & $\begin{array}{c}\text { MUERTE } \\
\text { MATERMA }\end{array}$ & $\begin{array}{c}\text { OTRAS } \\
\text { COMPLICACOMES }\end{array}$ \\
\hline UNO & 1.8 & 15.3 & -- & -- & 14.3 \\
DOS & 3.5 & 23.1 & 30.0 & 50.0 & 28.6 \\
$3-5$ & 43.9 & 30.8 & 20.0 & 50.0 & 33.3 \\
6 Y MAS & 30.8 & 30.8 & 50.0 & -- & 23.0 \\
\hline TOTAL & 100 & 100 & 100 & 100 & 100 \\
\hline
\end{tabular}

Tabla 20

TASA DE MORTALIDAD MATERNA

$\begin{gathered}\text { TASA DE MORTALIDAD } \\ \text { MATERNA DIRECTA }\end{gathered}=\frac{\begin{array}{c}\text { NUMERO DE MUERTES MATERNAS } \\ \text { PORARAZO, PARTO Y PUERPERIO }\end{array}}{\text { TOTAL DE NACIDOS VIVOS }} \times 100.000$

$$
T M M D=\frac{9}{3.245} \times 100.000=277
$$

siete $63.7 \%$. En los recién nacidos con pesos entre 1.500 y 2.499 el $9.5 \%$ tuvieron apgar a los cinco minutos menor de siete. En los recién nacidos entre 2.500 y 3.799 el $1.4 \%$ tuvieron apgar a los cinco minutos menor de siete. $\mathrm{Y}$ en los recién nacidos de 3.800 gramos o más el $1.3 \%$ tuvieron apgar a los cinco minutos menor de siete. Tabla 16.

TIPO DE COMPLICACION DEL RECIEN NACIDO SEGUN APGAR AL MINUTO

Las complicaciones del recién nacido que más se asociaron a apgar al minuto por debajo de siete fueron en su orden: el síndrome de dificultad respiratoria $79.3 \%$, recién nacido deprimido $42.3 \%$ y anomalías congénitas $36.3 \%$. Tabla 17 . TIPO DE COMPLICACIONES DEL RECIEN NACIDO SEGUN APGAR A LOS CINCO MINUTOS

Figura 12

TIPO DE PARTO

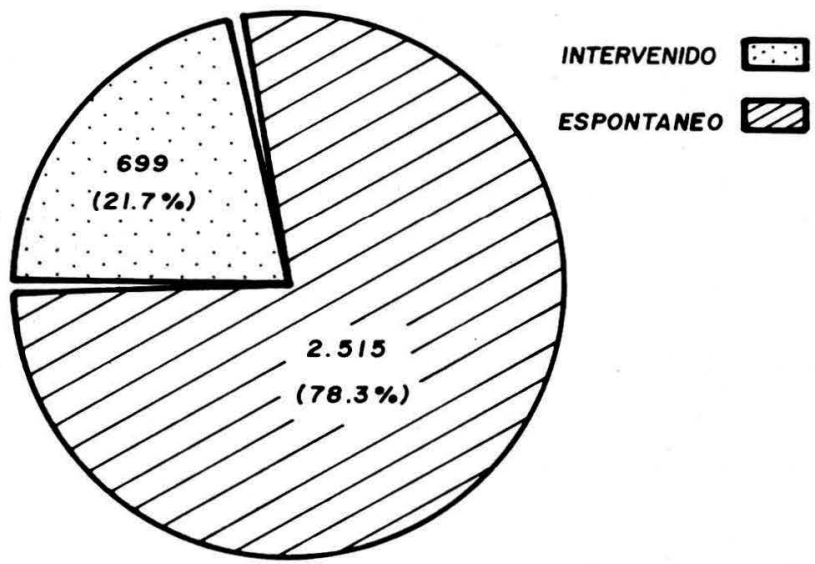

- NO APLICA 21 (ABORTOS)

- SIN INFORMACION 17
Figura 13

TIPO DE INTERVENCION

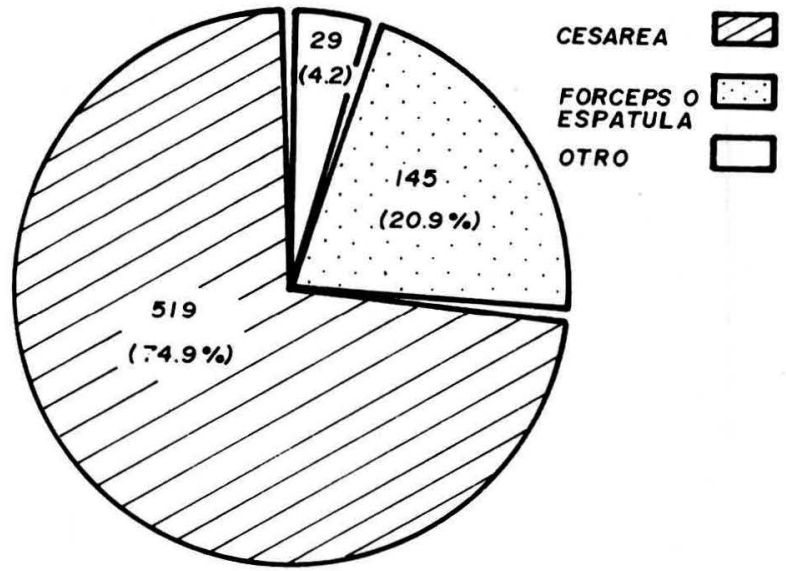

- NO INTERVENIDOS 2515

- NO APLICA 21 (ABORTOS)

- SIN INFORMACION 23

Las complicaciones del recién nacido que más se asociaron con apgar a los cinco minutos menor de siete fueron en su orden: el síndrome de dificultad respiratoria con $55.2 \%$, la infección con $20.0 \%$ y la macrosomía con $18.1 \%$. Tabla 18.

ESTANCIA HOSPITALARIA EN DIAS SEGUN COMPLICACION DEL PUERPERIO

Las complicaciones del puerperio con estancias hospitalarias de 3 y más días fueron en su orden la endometritis $94.7 \%$, otras infecciones $70.0 \%$ y hemorragia $61.6 \%$. Tabla 19.

TASA DE MORTALIDAD MATERNA (2)

Figura 14

COMPLICACION DEL PARTO

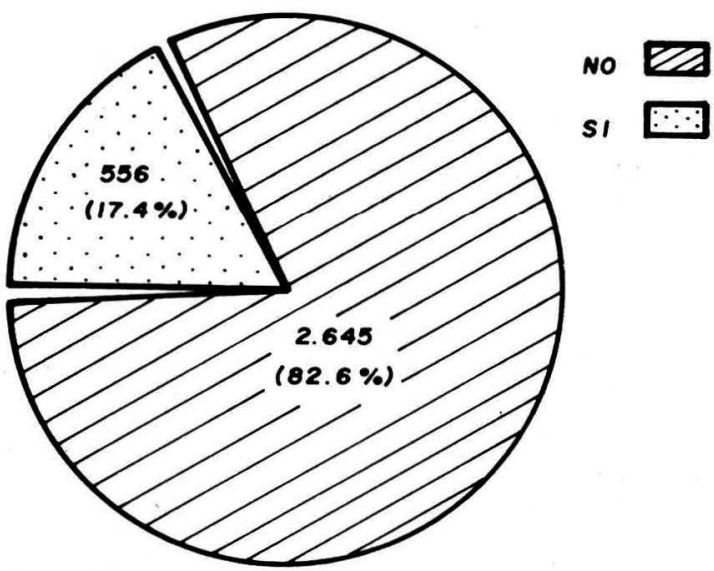

- SIN INFORMACION $5 I$. 
Durante el período del estudio nacieron vivos en las instituciones participantes 3.245 neonatos y hubo 9 muertes maternas lo que da una tasa de mortalidad materna de 277 x 100.000 nacidos vivos o 27.7 x 10.000 nacidos vivos. Tabla 20.

\section{Discusión de resultados}

Al observar la gran diferencia del porcentaje de embarazo en adolescentes entre el hospital con mayor incidencia $14.3 \%$ y el hospital eon menor incidencia $2.75 \%$, surge la duda de si en aquellas instituciones con incidencias muy bajas para nuestro medio $(4,8,10,14)$ no existirá un subregistro. Sin embargo la menor incidencia es explicable en las instituciones en las cuales la mayoría de pacientes son beneficiarias del ISS, ya que las adolescentes en Colombia con frecuencia carecen de los beneficios sociales (12). Tabla 1 .

En la distribución por edades se observa un incremento de pacientes a medida que aumenta la edad lo cual es de esperarse, pero con una estabilización del incremento a partir de los 17 años que hace suponer que en estos dos últimos grupos de mujeres adolescentes el comportamiento reproductivo es comparable a las mayores de 18. (Figura 1).

Prácticamente la mitad de las pacientes, $43.6 \%$, se encontraban sin el apoyo de una relación marital estable que viene a ser factor agravante de un embarazo considerado de alto riesgo (Figura 2). Si sumamos a este factor otros como la falta de control prenatal en más de la mitad de las pacientes, $58.2 \%$ (Figura 7) y cuando existe el control de una cuarta parte de los casos, $25.7 \%$, su número es insuficiente, sólo uno o dos controles (Figura 8).

El promedio de edad de aparición de la menarquia 12.8 años es similar a lo que reporta la literatura mundial $(1,14)$.

Es muy preocupante que el $26.3 \%$ de las pacientes tengan antecedentes de un parto (Figura 5); y que el 3.5\% tengan antecedente de aborto (Figura 6). Es decir, que casi una tercera parte de las adolescentes del estudio, $29.8 \%$, eran

Figura 15

TIPO DE COMPLICACIONES DEL PARTO

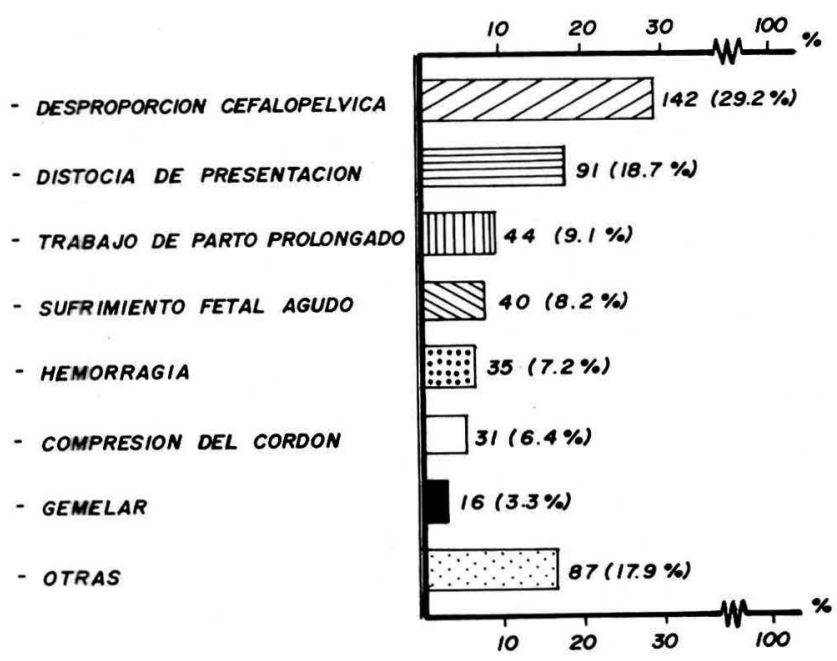

- SIN INFORMACION 70.
Figura 16

PESO DEL RECIEN NACIDO

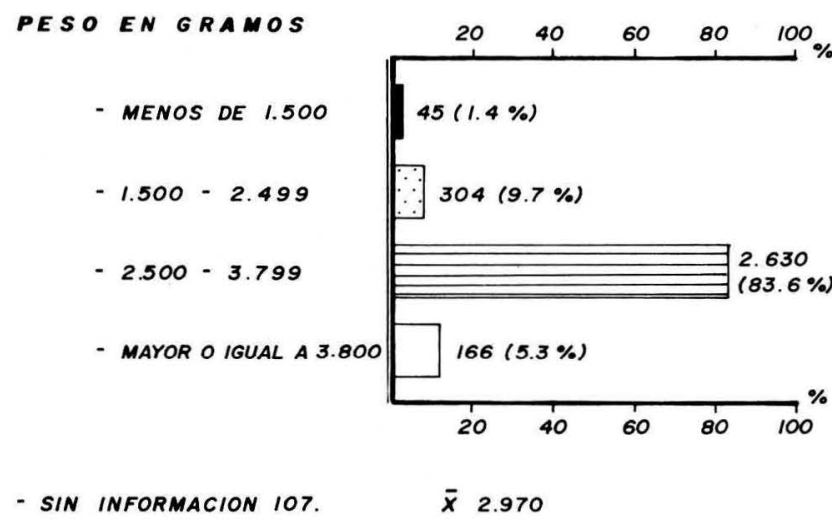

reincidentes en el embarazo lo que hace presumir una falla muy grande en la anticoncepción preventiva en el postparto y en el postaborto de las mujeres adolescentes en Colombia.

Casi la tercera parte de las pacientes, $28.7 \%$, presentaron morbilidad asociada al embarazo (Figura 9), siendo la principal patología la ruptura prematura de membranas, $28.4 \%$ (Figura 10). Las infecciones maternas que ocupan un segundo lugar dentro de la morbilidad asociada, la cérvicovaginitis y las enfermedades venéreas, se encontraron presentes en casi la mitad de las pacientes con morbilidad infecciosa, $46.0 \%$ (Figura 10A). Las infecciones cérvicovaginales y las sexualmente transmisibles se han relacionado con la ruptura prematura de membranas y ésta a su vez con el parto pretérmino que se encontró en el $21.5 \%$ de los casos (Figura 11); a su vez el parto pretérmino se asocia con el bajo peso al nacer que se encontró en el $11.1 \%$ (Figura 16). Dentro de las afecciones del recién nacido la principal morbilidad fue la prematurez con $50.2 \%$ (Figura 19).

Figura 17-A

APGAR AL MINUTO

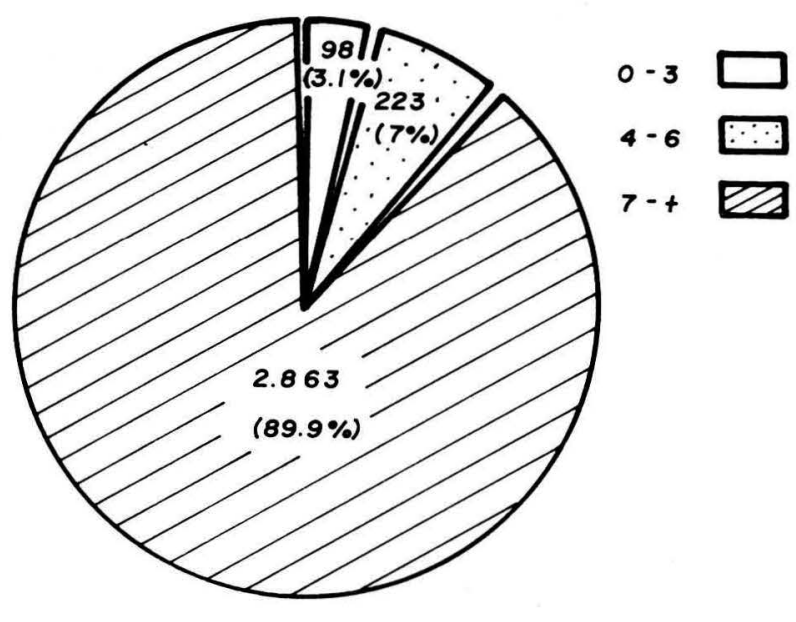

- SIN INFORMACION 68. 
Figura 17-B

\section{APGAR A LOS 5 MINUTOS}

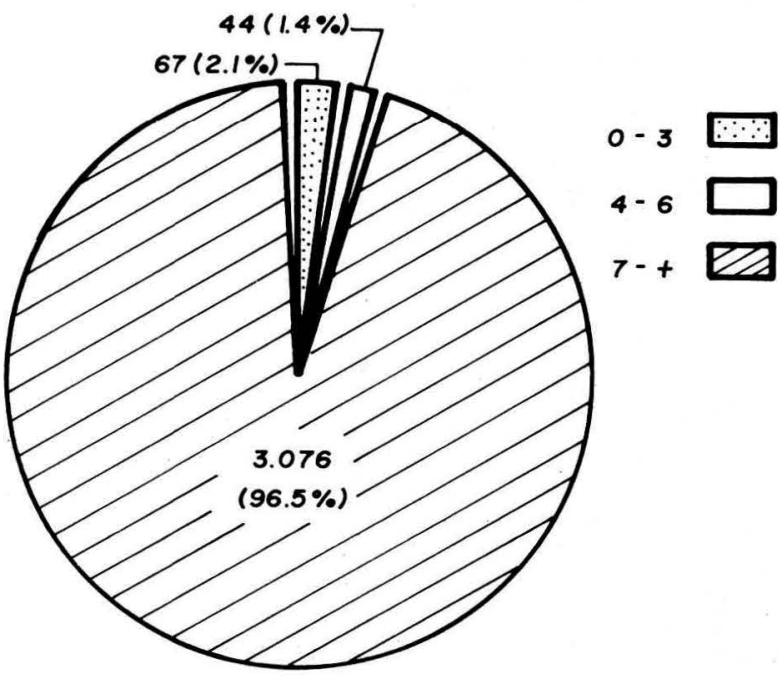

- SIN INFORMACION 65

Casi la cuarta parte de los partos fueron intervenidos, $21.7 \%$ (Figura 2); y en la gran mayoría la intervención practicada fue la cesárea, $74.9 \%$ (Figura 13). La cesárea se relaciona directamente con el tipo de complicación del parto que indicó el procedimiento obstétrico a seguir: desproporción cefalopélvica, distocia de presentación y embarazo gemelar (Figura 15).

La principal complicación del puerperio fue la endometritis, $67.0 \%$ (Figura 21); y a su vez la responsable de la mayor estancia hospitalaria $43.9 \%$ con 3 a 5 días y $50.8 \%$ con 6 y más días (Tabla 19).

La principal morbilidad asociada al embarazo en las pacientes de 15 años fue la hipertensión $25.6 \%$, ocupando en

Figura 18

\section{PATOLOGIA DEL RECIEN NACIDO}

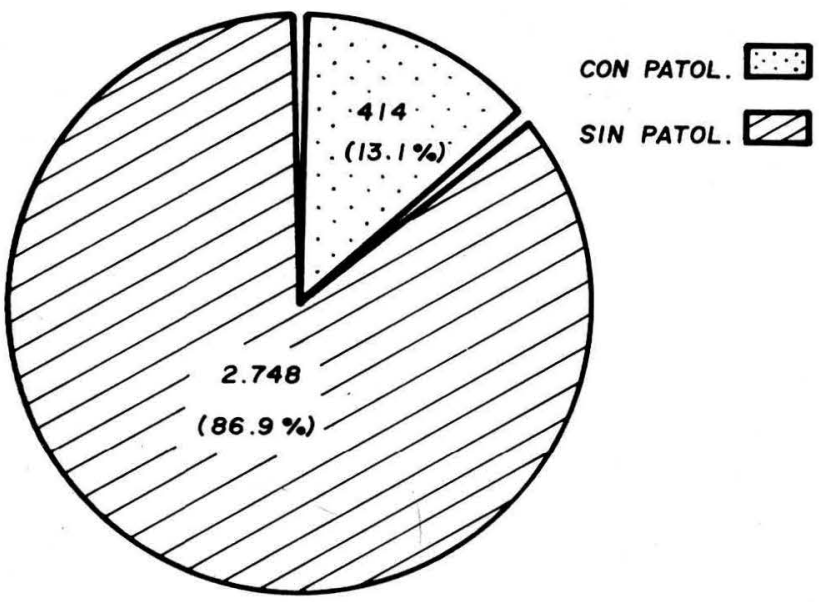

- SIN INFORMACION 90. este grupo el primer lugar; en los demás grupos de edad la hipertensión ocupó el tercer lugar (Tabla 2).

Las pacientes sin control prenatal tuvieron siempre mayor morbilidad asociada al embarazo en todas las patologías (Tabla 4); mayor parto intervenido (Tabla 5); mayor complicación del parto en todo tipo de complicación (Tabla 6); mayor complicación en el puerperio y las dos únicas muertes maternas en este período (Tabla 7). Lo anterior viene a demostrar la importancia que el control prenatal tiene y el mayor riesgo a que se exponen las pacientes que no acuden a esta importante consulta.

La morbilidad asociada al embarazo que más se asoció con recién nacido de bajo peso, $42.6 \%$, fue la amenaza de parto pretérmino (Tabla 9); y la mayor estancia hospitalaria fue por la distocia, $76.2 \%$, con más de tres días (Tabla 10). Estas dos patologías tienen una clara relación de causa efecto, la amenaza del parto pretérmino con el parto prematuro y el recién nacido de bajo peso; y la distocia con la cesárea.

Las complicaciones del puerperio fueron más frecuentes en las pacientes intervenidas con cesárea que con fórceps (Tabla 11) y como consecuencia la mayor estancia hospitalaria la tuvieron las pacientes intervenidas con cesárea $64.4 \%$ con 3 o más días (Tabla 12).

La complicación del parto que con más frecuencia se asoció con apgar al minuto de 6 o menos fue el sufrimiento

Figura 19

TIPO DE PATOLOGIA DEL RECIEN NACIDO

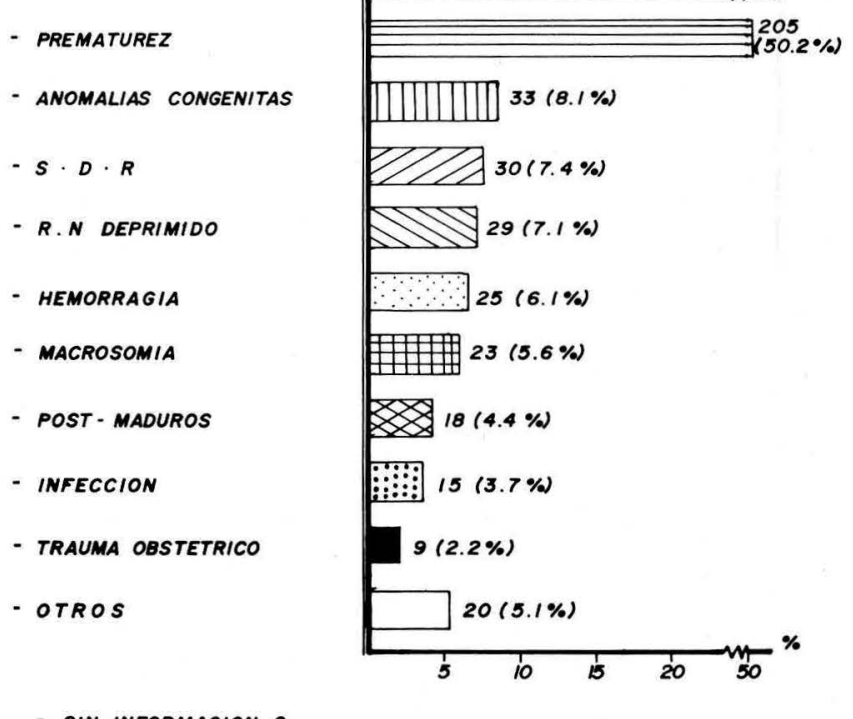

- SIN INFORMACION 6.

fetal agudo con $35.9 \%$ (Tabla 13); y la complicación del parto que con más frecuencia se asoció con apgar a los cinco minutos de 6 o menos fue la compresión del cordón con 12.9\% (Tabla 14). Esta asociación se explica fácilmente por la hipoxia intraparto que en ambas entidades se presenta.

Los recién nacidos de menos de 1.500 gramos tuvieron en su gran mayoría apgar al minuto de 6 o menos, $79.5 \%$ (Tabla 15), y a los cinco minutos permanecían con apgar de 6 o menos el $63.7 \%$ (Tabla 16). Esto viene a demostrar 
Figura 20 COMPLICACION DEL PUERPERIO

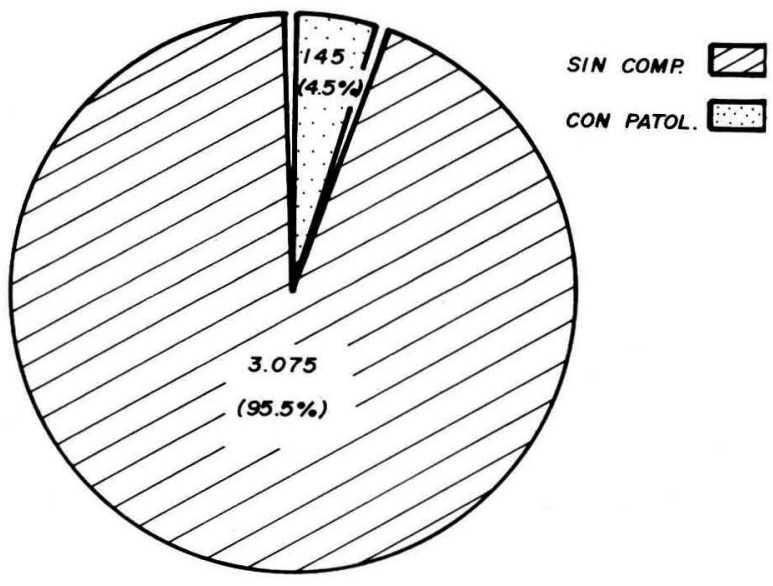

- SIN INFORMACION 32.

el altísimo riesgo que un peso menor de 1.500 gramos al nacer expone al recién nacido a no tener una buena calidad de vida futura.

La complicación del recién nacido que con más frecuencia se asoció con apgar de 6 o menos fue el síndrome de dificultad respiratoria, tanto en la calificación al minuto, $79.3 \%$, como en la calificación a los 5 minutos, 55.2\% (Tablas 17 y 18). A su vez el síndrome de dificultad respiratoria se asocia directamente con el parto pretérmino y el recién nacido prematuro.

La tasa de mortalidad materna encontrada en el estudio de 277 x 100.000 nacidos vivos, es muy superior si se compara con la tasa oficial del Ministerio de Salud para todas las edades en 1988 que es de $100 \times 100.000$ nacidos vivos (9); o con la tasa de mortalidad materna para todas las edades en un estudio colaborativo interinstitucional realizado en 1979, que fue de 232 x 100.000 nacidos (15); y aún ligeramente superior a la tasa de mortalidad materna para Latinoamérica que en 1983 fue de 270 x 100.000 nacidos vivos y exageradamente superior si se compara con la de los países desarrollados en el mismo período que fue de $30 \times 100.000$ nacidos vivos (6).

\section{Conclusions}

The absence of permanent partner is a very frequent risk factor at this age.

The appearance average of the menarche was of 12.8 years.

Failure in the preventive contraception in the postpartum or postabortion.

Absence of prenatal control in the majority of patients and deficient service in a great proportion when it exists.

Morbidity associated to pregnancy, childbirth and puerperium complications were more frequent in the group of patients without prenatal control.

The childbirth complication which caused longer staying at hospital was the distocia.

The more frequent childbirth intervention was the cesarea, which had also puerperial morbidity, and as a consequence of it a longer staying at hospital.

The childbirth complication which more frequently produced qualifications of apgar at a minute minor than 7 , was the intradelivery acute fetal suffering; and qualifications of apgar at five minutes minor than 7 , was the umbilical cord compression.

The more frequently newborn complication associated with apgar minor than 7 at minute as weill as five minutes was the respiratory syndrome difficulty.

The maternal mortality rate was $277 \times 100.000$ births alive.

\section{Conclusiones}

La ausencia de compañero permanente es un factor de riesgo muy frecuente en esta edad.

El promedio de aparición de la menarquia fue de 12.8 años.

Falla en la anticoncepción preventiva en el postparto o postaborto.

Control prenatal ausente en la mayoría de las pacientes y cuando existe, en una proporción importante éste es deficiente.

La morbilidad asociada al embarazo, las complicaciones del parto y las complicaciones del puerperio fueron más frecuentes en el grupo de pacientes sin control prenatal.

La complicación del parto que ocasionó mayor estancia hospitalaria fue la distocia.

La intervención del parto más frecuente fue la cesárea, que a su vez tuvo con más frecuencia morbilidad puerperal y como consecuencia mayor estancia hospitalaria.

La complicación del parto que con mayor frecuencia produjo calificaciones de apgar al minuto menores de 7 , fue el sufrimiento fetal agudo intraparto; y calificaciones de apgar a los cinco minutos menores de 7, la compresión del cordón.

La complicación del recién nacido que se asoció con mayor frecuencia con apgar menores de 7 tanto al minuto como a los cinco minutos fue el síndrome de dificultad respiratoria.

La tasa de mortalidad materna fue de $277 \times 100.000$ nacidos vivos.

Figura 21

TIPO DE COMPLICACION PUERPERAL

- ENDOMETRITIS

- HEMORRAGIA

- otRAS INFECcIONES

- MUERTE MATERNA

OTRAS COMPLICACIONES

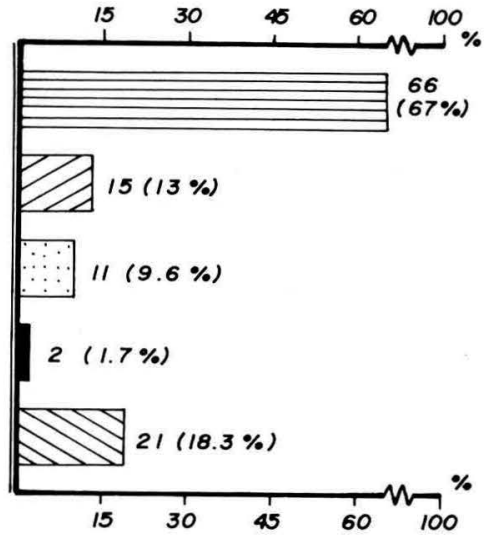




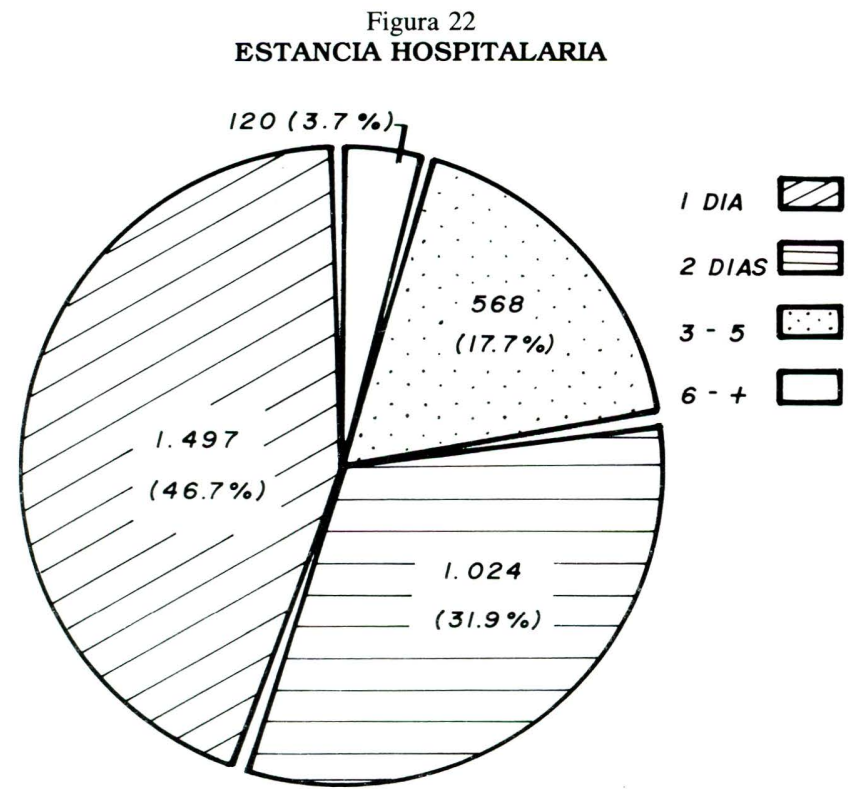

- SIN INFORMACION 43.

$\bar{x} \quad 1.74$ DIAS

\section{Recomendaciones}

Proporcionar suficientes servicios de anticoncepción a los adolescentes a fin de prevenir el embarazo en este período de la vida ya que su altísimo riesgo ha quedado plenamente demostrado en las conclusiones del estudio.

Implementar en las instituciones que atiendan partos en Colombia servicios de atención prenatal, parto y postparto dirigidos especialmente para la adolescente embarazada.

Promover, continuar e intensificar la educación sexual en los currículos de los colegios desde el primer año de secundaria.

Preparar mejor al personal de salud: médicos, enfermeras, psicólogos, trabajadores sociales, etc., modificando los currículos universitarios para que incluyan o intensifiquen componentes de salud reproductiva de educación sexual y planificación familiar con el propósito de que en el futuro en su ejercicio profesional puedan prestar una adecuada atención a los adolescentes en Colombia.

\section{Agradecimientos}

- Al Doctor Ricardo Vernón, Director del Population Council Colombia, por su asesoría en el procesamiento de la información.

- A Profamilia Bogotá, por facilitar el computador para el procesamiento de datos.

- A todos y cada uno de los investigadores institucionales, ya que sin su colaboración en la recolección de la información no hubiese sido posible realizar la presente investigación.

\section{BIBLIOGRAFIA}

1. BALlarD, E. "Aspectos Médicos y de Salud de la Reproducción en la.Adolescente”. Clínicas Obstétricas y Ginecológicas, junio 1971.

2. CENTRO LATINOAMERICANO DE PERINATOLOGIA Y DESARROLLO HUMANO (CLAP) - OPS - OMS. "Salud Perinatal", vol. 2, No. 4, pág. 32 de 1985.

3. DELGADO, BIENVENIDO. "Peligro del embarazo en la adolescencia”, Boletín Asociación Dominicana Probienestar de la Familia Inc. mayo-junio de 1977, vol. 9, No. 81 .

4. DUARTE CONTRERAS, ALBERTO. "El embarazo en adolescentes es de alto riesgo". Revista Colombiana de Obstetricia y Ginecología 1975; vol. XXVI, No. 6: 385-95

5. ELSTER, ARTHUR; PANZARINE, SUSAN; McANARNEY, ELIZABETH. "Causes of Adolescent Pregnancy". Medical Aspect of Human Sexuality, vol. 14, No. 7, July 1980.

6. FAMILY HEALTH INTERNATIONAL (FHI). "Maternal Mortality is Global Problem”. Network, vol. 9, No. 1, pág. 3, Sall 1987.

7. FLICK, L. H. "Paths to Adolescent Parenthood: Implications for prevention". Public Health Rep. Mar-Apr 1986; 101(2): 132-47

8. LOPEZ, G.; RIAÑO, G.; STEWART, K. "La embarazada adolescente", Monografías CCRP. Avances en Obstetricia y Ginecología, área biomédica, vol. 14, sept. de 1979.

9. MINSALUD (Dirección de Atención Médica - División Materno
Infantil y Dinámica de Población). "Diagnóstico de salud reproductiva en Colombia", 1988, pág. 23.

10. MORENO, ALBERTO. "Adolescentes y Embarazo", Revista Colombiana de Obstetricia y Ginecología, vol. 39, No. 3 (235-44). julio-septiembre de 1989

11. PERLMAN, S.B.; KLERMAN, L.V.; KINARD, E.M. "The use of socioeconomic data to predict teenage birth rates. An exploratory study in Massachusetts". Public Health Rep. Jul-Aug 1981; 96(44): 335-41.

12. RICO DE ALONSO, ANA. "Madres solteras adolescentes". Plaza y Janes. Primera Edición, febrero de 1986.

13. TORRES DE HERING, LUCY. "Adolescencia en Colombia. Catálogo colectivo de materiales bibliográficos". Asociación Salud con Prevención. Bogotá, febrero de 1987.

14. URIZA, GERMAN. "Embarazo en Adolescentes". Revista Colombiana de Obstetricia y Ginecología, vol. XXXIV, No. 2: 102-14. marzo-abril de 1983.

15. URIZA, GERMAN; LOPEZ, GUILLERMO; RIAÑO, GERMAN; ESTRADA, ALCIDES. "Estudio Hospitalario de Mortalidad Materna". Revista Colombiana de Obstetricia y Ginecología, vol. 23, No. 5 (325-42), septiembre-octubre 1982. 